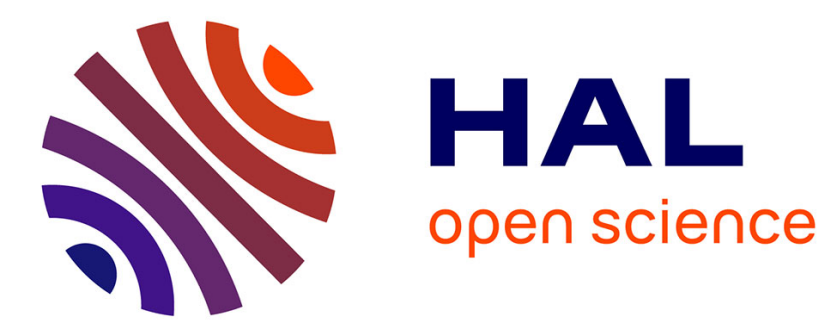

\title{
Modification de la loi de Charlesby. I. Calcul du profil de la densité de coupure créée dans une couche de polymère par des faisceaux de particules
}

A. Schiltz, Agnès Weill, P. Paniez

\section{- To cite this version:}

A. Schiltz, Agnès Weill, P. Paniez. Modification de la loi de Charlesby. I. Calcul du profil de la densité de coupure créée dans une couche de polymère par des faisceaux de particules. Revue de Physique Appliquée, 1984, 19 (6), pp.431-438. 10.1051/rphysap:01984001906043100 . jpa-00245213

HAL Id: jpa-00245213

https://hal.science/jpa-00245213

Submitted on 1 Jan 1984

HAL is a multi-disciplinary open access archive for the deposit and dissemination of scientific research documents, whether they are published or not. The documents may come from teaching and research institutions in France or abroad, or from public or private research centers.
L'archive ouverte pluridisciplinaire HAL, est destinée au dépôt et à la diffusion de documents scientifiques de niveau recherche, publiés ou non, émanant des établissements d'enseignement et de recherche français ou étrangers, des laboratoires publics ou privés. 


\title{
Modification de la loi de Charlesby. I. Calcul du profil de la densité de coupure créée dans une couche de polymère par des faisceaux de particules
}

\author{
A. Schiltz, A. Weill et P. Paniez \\ C.N.E.T., B.P. 98, Chemin du Vieux Chêne, 38243 Meylan Cedex, France
}

(Reçu le 16 janvier 1984, accepté le 15 mars 1984)

\begin{abstract}
Résumé. - A partir de la courbe de développement d'une couche de " résine positive ", on calcule le profil de la densité de coupure le long de l'épaisseur irradiée. Si l'on connaît le taux de dégradation, $G$, on peut calculer le profil de l'énergie effective déposée le long de l'épaisseur - et réciproquement. Nous appelons énergie effective, l'énergie déposée qui provoque effectivement une modification du polymère. Nous appliquons la loi de Charlesby décrivant la relation entre la dose et la masse moléculaire moyenne en nombre des chaînes macromoléculaires fragmentées à deux exemples pris dans la littérature (électrons/20 keV/PMMA et protons/50 keV/PMMA).

Nous montrons que la loi de Charlesby est une loi locale qui devrait permettre de calculer la masse moléculaire moyenne mesurable par GPC sur toute l'épaisseur irradiée lorsque l'énergie déposée est constante sur toute l'épaisseur irradiée.
\end{abstract}

Nous constatons que la relation est linéaire mais qu'elle n'est cependant pas vérifiée à dose nulle extrapolée.

\begin{abstract}
For positive resists, the scission density profile versus irradiated depth is based on the development curve. If the value, $G$ for scission yield is known, the profile of the effective energy absorbed in the depth can be calculated - and inversely. The effective energy is taken as the absorbed energy required to actually modify the polymer. In this paper, Charlesby's law, which links the particle dose to the average molecular weight, is used to analyse two cases taken from the litterature : electrons bombarded at $20 \mathrm{keV}$ on PMMA and protons bombarded at $50 \mathrm{keV}$ on PMMA.

Charlesby's law proves to be a local law. When the absorbed energy remains constant along the irradiated depth, the law is assumed to yield the same molecular weight as that obtained from GPC analysis of the entire layer.

Charlesby's relation is proved to be linear, but is not in agreement with the extrapolated zero-dose value.
\end{abstract}

\section{Généralités.}

Le principe de la modification des polymères sous l'effet des bombardements ionique et électronique est aujourd'hui connu $[1,2]$.

Lorsqu'ils pénètrent dans les polymères, les ions et les électrons ne perdent pas leur énergie de la même façon : en effet, les ions pénètrent peu profondément et déposent beaucoup d'énergie avec une dispersion faible alors que les électrons pénètrent profondément et déposent peu d'énergie avec une dispersion élevée [3].

La masse moléculaire moyenne en nombre des chaînes fragmentées, $\overline{M n}, f$, dans le cas des « résines positives » (ou réticulées, dans le cas des « résines négatives $"$ ) est fonction des trois paramètres suivants :

- La dose incidente, exprimée en flux de charge électrique, $D\left(\mathrm{C} / \mathrm{cm}^{2}\right)$, ou en flux de particules, $Q$ (nombre de particules $/ \mathrm{cm}^{2}$ ).

- L'énergie déposée par une particule et par unité d'épaisseur, $E_{\mathrm{d}}(\mathrm{eV} / \mathrm{nm})$.
- Le taux de coupure du matériau, $G$, exprimé en nombre d'événements par $100 \mathrm{eV}$ absorbés.

Les effets observables de l'irradiation sont la fragmentation et la réticulation des chaînes macromoléculaires, ce qui provoque une variation de la solubilité du polymère. Dans le cas d'une résine positive, le taux de dégradation, $G_{\mathrm{s}}$, est supérieur au taux de réticulation, $G_{x}$, bien que celui-ci ne soit pas nul [1].

Dans le cas des ions comme dans celui des électrons, il est souvent admis que l'énergie déposée par unité de longueur, $E_{\mathrm{d}}$, est constante dans toute l'épaisseur irradiée.

Cela est raisonnable lorsque l'épaisseur irradiée est faible devant le parcours moyen projeté, $R_{\mathrm{p}}\left({ }^{1}\right)$, des particules. Cependant, lorsque l'épaisseur est voisine ou supérieure à $R_{\mathrm{p}}$, l'énergie déposée est une fonction qui varie fortement autour de $R_{\mathrm{p}}$ (Fig. 1).

$\left({ }^{1}\right)$ Défini dans les notations. 


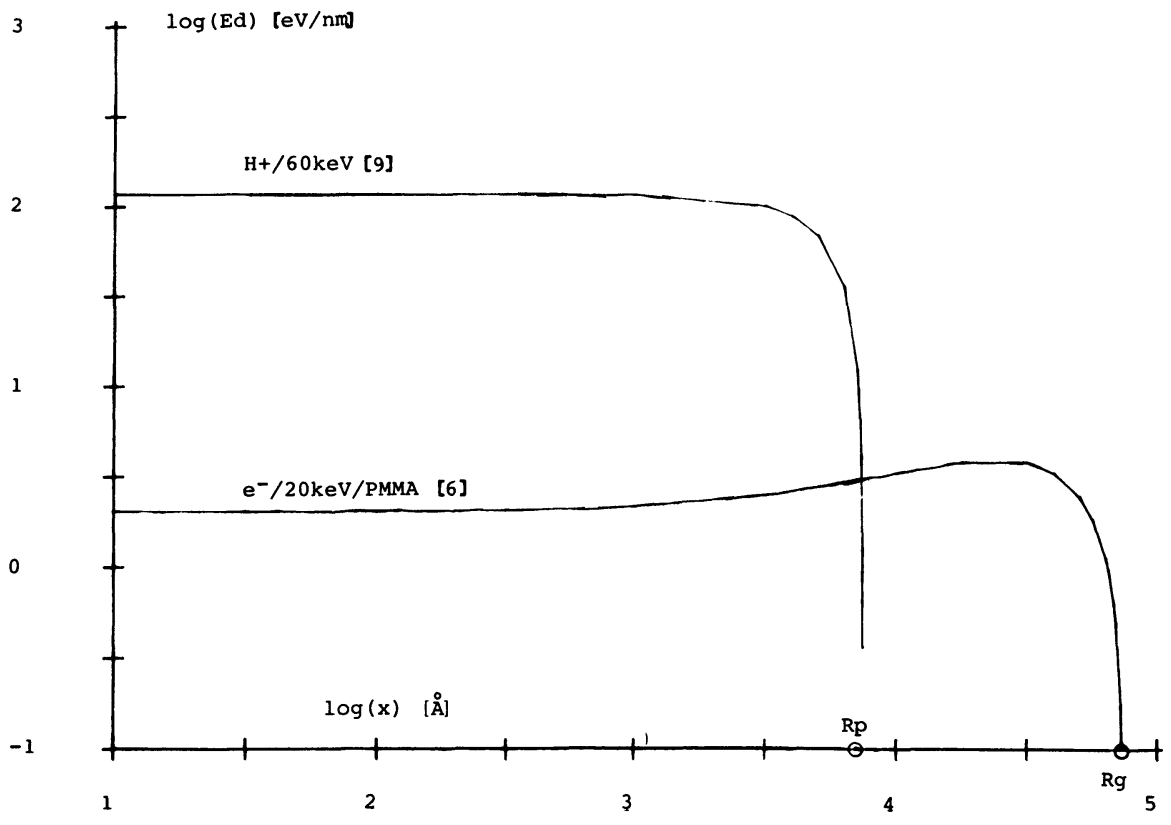

Fig. 1. - Profils théoriques de l'énergie déposée.

[Theoretical profiles for the energy deposition.]

Enfin, on admettra, partout où intervient l'expression théorique de $E_{\mathrm{d}}$, l'hypothèse que les phénomènes de diffusion et de rétrodiffusion ont un effet négligeable.

\section{- esure \\ à partir des courbes de développement.}

A partir de théories de physique nucléaire, des formules ont été proposées qui donnent accès au taux linéaire de dégradation, $\left(G \cdot E_{\mathrm{d}}\right)(x)$, et qui permettent de prévoir les courbes de développement [4].

Nous proposons une méthode expérimentale simple pour mesurer ce taux de dégradation à partir des courbes de développement.

partir une cour e ve oppem , $x \quad\left(^{2}\right)$,

$\left({ }^{2}\right)$ Défini dans les notations.

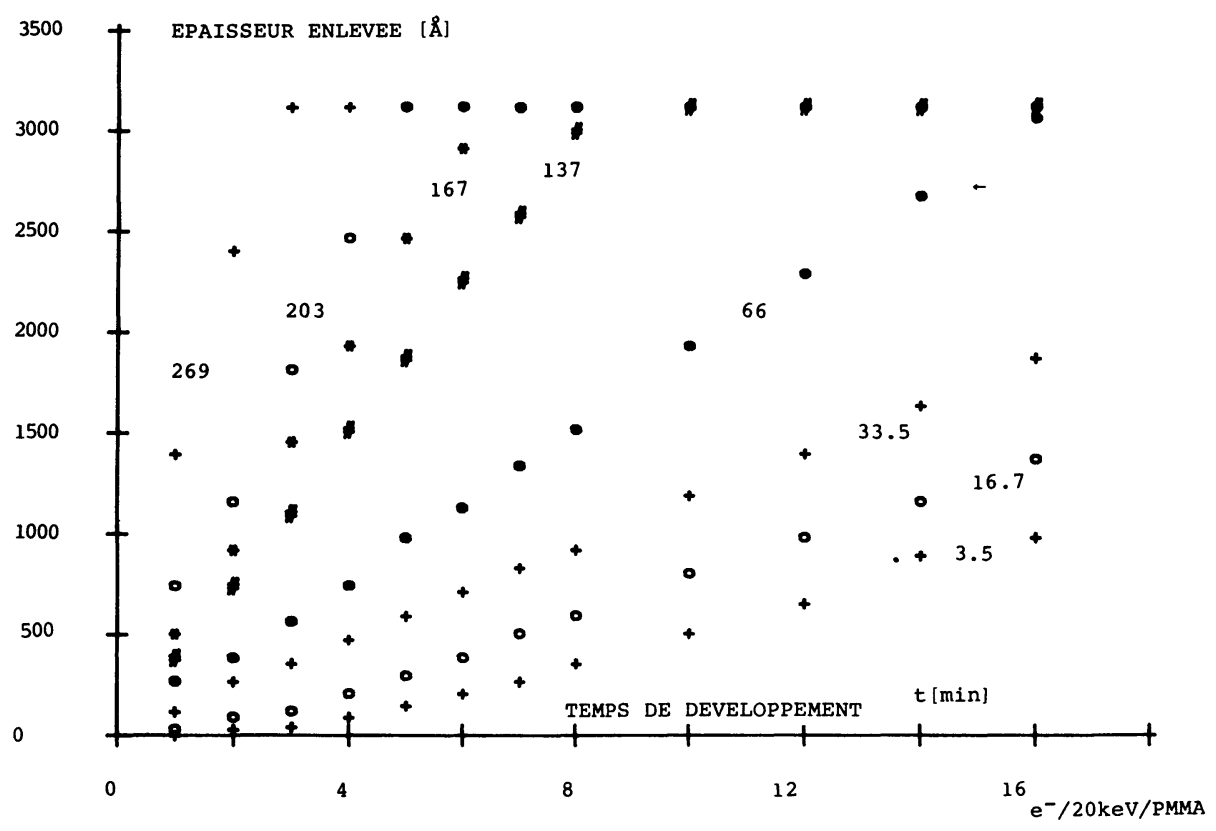

Fig. 2. - Epaisseur enlevée $x(t)$ d'après [5]. Chiffres : dose $\left[10^{12} \mathrm{el} / \mathrm{cm}^{2}\right]$.

[Removed thickness $x(t)$ from [5].] 


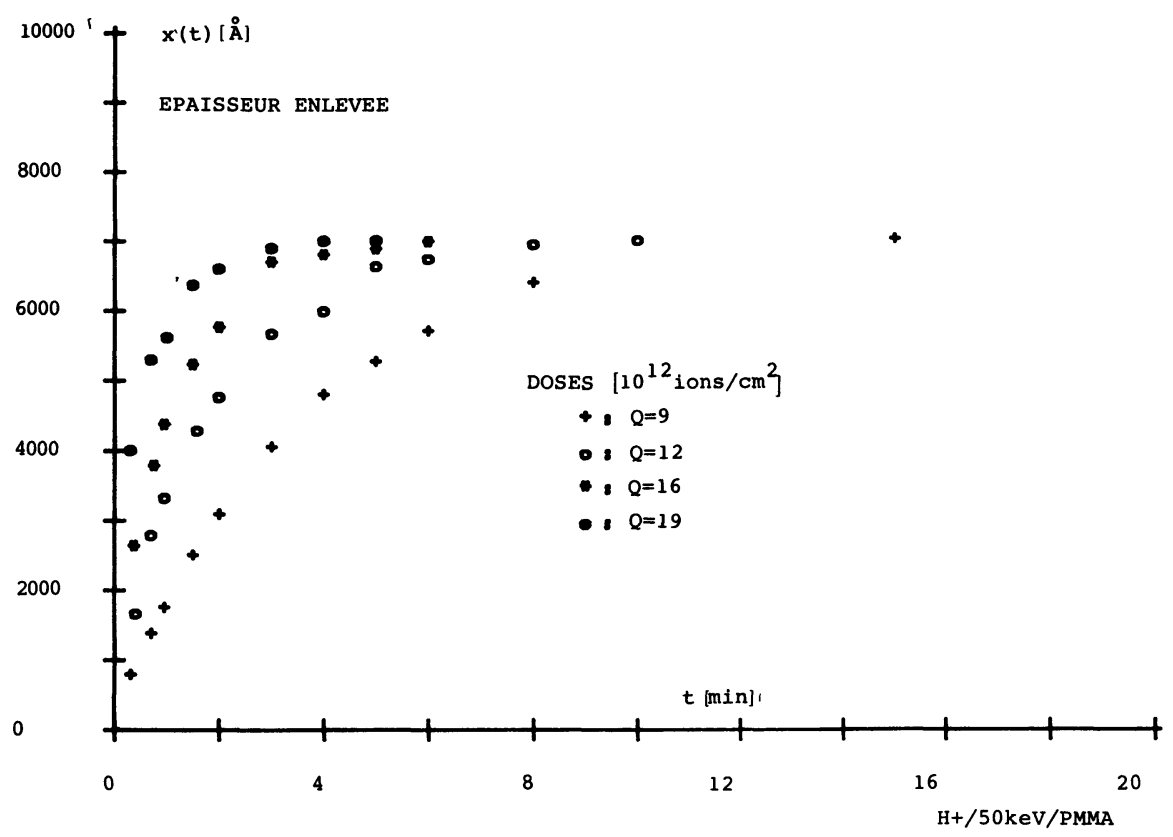

Fig. 3. - Epaisseur enlevée $x(t)$ d'après [7].

[Removed thickness $x(t)$ from [7].]

comme celles des figures 2 et 3 , on obtient le profil de vitesse de dissolution, $V_{\mathrm{d}}(x)$.

A l'aide de la loi de dissolution de Greeneich [5] :

$$
V_{\mathrm{d}}(\overline{M n})=A+B \cdot(1 / \overline{M n})^{(\alpha)}
$$

on calcule le profil des masses fragmentées, $\overline{M n}, f(x)$. On utilise la loi de Charlesby [1, 2] en supposant qu'elle s'applique localement, c'est-à-dire en tout point, $x$, de l'épaisseur irradiée, et en supposant que la dose est constante dans l'épaisseur irradiée $\left(e<R_{\mathrm{p}}\right)$ :

$$
1 / \overline{M n}, f(x)=\frac{1}{\overline{M n}, o}+\frac{G \cdot Q \cdot E_{\mathrm{d}}(x)}{100 \rho \cdot N_{\mathrm{A}}} .
$$

Dès lors, connaissant $\overline{M n}, f(x)$, on obtient expérimentalement le profil de la densité de dégradation, $\left(G . Q . E_{\mathrm{d}}\right)(x)$. Elle s'exprime en nombre de coupures effectives par $\mathrm{nm}^{3}$.

On obtient également (Fig. 4) le taux linéaire de dégradation, $\left(G . E_{\mathrm{d}}\right)(x)$, à l'aide de (3) :

$\left(G \cdot E_{\mathrm{d}}\right)(x)=\frac{100 \rho \cdot N_{\mathrm{A}}}{Q} \cdot\left[\left(\frac{V_{\mathrm{d}}(x)-A}{B}\right)^{1 / \alpha}-\frac{1}{\overline{M n}, o}\right]$.

Cette relation permet, connaissant le taux de coupures, $G$, de tracer $E_{\mathrm{d}}(x)$ et réciproquement, de calculer $G$ lorsqu'on connaît le profil théorique de l'énergie déposée.

Enfin, il convient de remarquer que la valeur mesurée de $\left(G \cdot E_{\mathrm{d}}\right)(x)$ obtenue à partir des courbes expérimentales de développement, permet de prévoir les courbes de développement qui seraient obtenues dans des conditions expérimentales différentes. Pour cela, il suffit d'appliquer la démarche inverse de celle exposée ci-dessus. Elle conduit à l'équation (4) :

$$
t(e)=\int_{0}^{e} \frac{\mathrm{d} x}{A+B \cdot\left[\frac{1}{\overline{M n}, o}+\frac{\left(G \cdot E_{\mathrm{d}}\right)(x) \cdot Q}{100 \rho \cdot N_{\mathrm{A}}}\right]^{\alpha}} .
$$

2. Calcul de la masse moyenne de la couche dégradée. L'équation (2) suppose que la loi de Charlesby est applicable localement en tout point $x$ de l'épaisseur irradiée.

Si la loi de Charlesby était applicable globalement, c'est-à-dire sur toute l'épaisseur, $e$, de la couche, on devrait pouvoir écrire aussi :

$$
\frac{1}{\overline{M n, f, T^{(e)}}}=\frac{1}{\overline{M n}, o}+\frac{G \cdot Q \cdot \bar{E}_{\mathrm{d}}^{(e)}}{100 \rho N_{\mathrm{A}}}
$$

où $\overline{M n, f, T^{(e)}}$ est la masse moléculaire moyenne totale de la résine, intégrée sur toute la couche d'épaisseur $e$ :

$$
\overline{M n, f, T^{(e)}}=\frac{\int_{0}^{e} \overline{M n}, f(x) \cdot \mathrm{d} x}{\int_{0}^{e} \mathrm{~d} x} .
$$

Expérimentalement, cette masse moyenne est celle qui est mesurée par Chromatographie par Perméation 


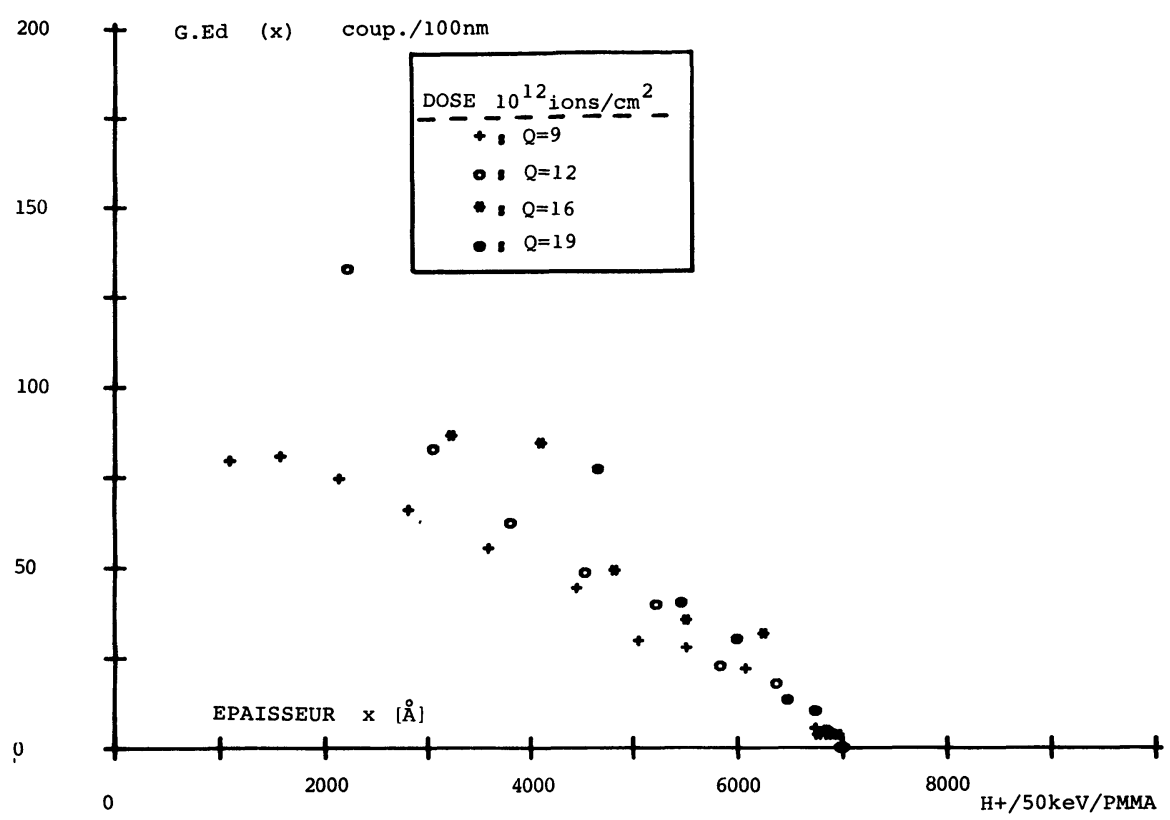

Fig. 4. - Taux linéaire de dégradation.

[Linear scission yield.]

de Gel (GPC) car toute la couche de résine participe à la mesure; et où $\bar{E}_{\mathrm{d}}^{(e)}$ est l'énergie moyenne déposée dans la couche $e$ :

$$
\bar{E}_{\mathrm{d}}^{(e)}=\frac{0{ }^{x \cdot \mathrm{d} x}}{\int_{0}^{e} \mathrm{~d} x} .
$$

Or, en utilisant (2), on obtient en réalité :

$$
\frac{1}{\overline{M n, f, T^{(e)}}}=\frac{e}{\int_{0}^{e} \frac{\mathrm{d} x}{\frac{1}{\overline{M n}, o}+\frac{G \cdot Q \cdot E_{\mathrm{d}}(x)}{100 \rho \cdot N_{\mathrm{A}}}}}
$$

Bien entendu, (6) n'est pas équivalente à (5), sauf lorsque l'énergie déposée est constante le long de $x$.

Pour illustrer ce propos, définissons un profil linéaire théorique de $E_{\mathrm{d}}(x)$ :

$$
E_{\mathrm{d}}=p \cdot(1-x / r) \quad p \text { et } r \text { étant constants } .
$$

Sur la figure 5 sont reportés les graphes des fonctions $1 / \overline{M n, f, T^{(e)}}=f(Q)$ obtenus avec l'équation (5) et avec l'équation (6). On voit que les deux graphes sont distincts.

D'autre part, on observe, dans le cas de l'équation (6), que la variation de $1 / \overline{M n, f, T^{(e)}}=f(Q)$ n'est pas linéaire. On en conclut donc que la loi de Charlesby ne peut pas s'appliquer globalement sur toute l'épaisseur lorsque l'énergie déposée varie le long de l'épaisseur $x$.

\section{Application à deux exemples ( $\left.\mathrm{e}^{-} / 20 \mathrm{keV} / \mathrm{PMMA}\right)$ et $\left(\mathrm{H}^{+} / 50 \mathrm{keV} / \mathrm{PMMA}\right)$}

Nous avons étudié les deux cas $e \ll R_{\mathrm{p}}$ et $e>R_{\mathrm{p}}$ en appliquant le traitement décrit en I.A. aux deux exemples suivants.

3.1 Application au CAS ÉlectroniQue $\left(\mathrm{e}^{-} / 20 \mathrm{keV} /\right.$ $\left.e \ll R_{\mathrm{p}}\right)$. - Les courbes de développement et la loi de dissolution sont tirées de [5]. Elles concernent un PMMA $\left(\overline{M n}, o \simeq 200000 ; \rho=1,19 \mathrm{~g} / \mathrm{cm}^{3}\right)$ irradié par des électrons d'une énergie incidente de $20 \mathrm{keV}$ et développé dans la Méthyl-Isobutyl-Cétone (MIBK) après un recuit de $90 \mathrm{~min}$. à $160^{\circ} \mathrm{C}$.

Les paramètres de dissolution sont :

$$
\begin{aligned}
& A=84 \AA / \mathrm{min} \\
& B=3,14 \times 10^{8} \\
& \alpha=1,5 .
\end{aligned}
$$

L'épaisseur initiale, environ $3000 \AA$, est faible devant le parcours moyen projeté (GRUN Range, [6]) : $R_{\mathrm{G}}=73116 \AA$. L'énergie déposée vaut selon [6] (Fig. 1) :

$$
E_{\mathrm{d}}(x)=2,7354\left(0,74+4,7 z-8,9 z^{2}+3,5 z^{3}\right)
$$

avec

$$
z=x / R_{\mathbf{G}}
$$

Elle varie relativement peu sur l'intervalle d'épaisseur irradiée et nous allons donc la considérer comme constante :

$$
\bar{E}_{\mathrm{d}}=2,3 \pm 0,3 \mathrm{eV} / \mathrm{nm}
$$




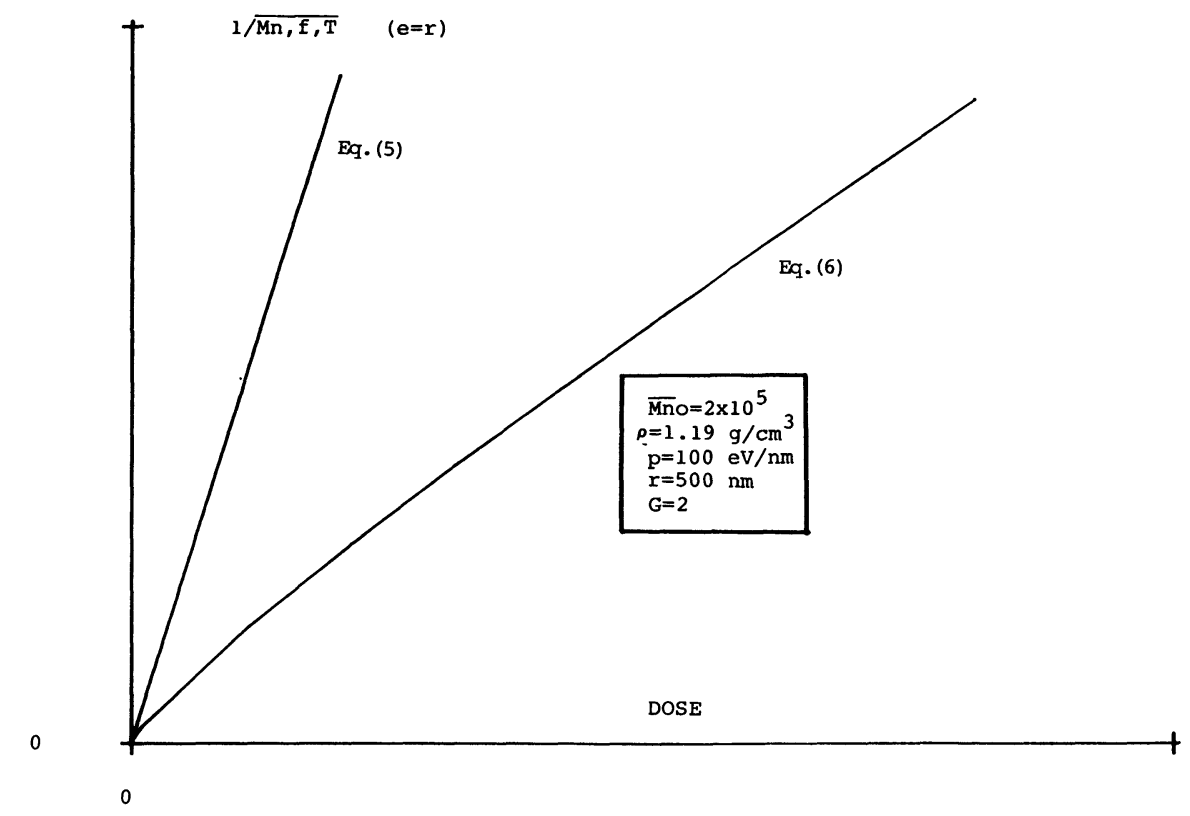

Fig. 5. - Graphes de $1 / \overline{M n, f, T}=f(Q)$.

[Curves for $1 / \overline{M n, f, T}=f(Q)$.]

Comme $\bar{E}_{\mathrm{d}}$ est une constante, $\overline{M n}, f$ et $V_{\mathrm{d}}$ sont également des constantes et les courbes de développement sont des droites de pente $V_{\mathrm{d}}$ ne dépendant que de la dose. C'est bien ce qu'on observe figure 2. Dans ce cas la loi de Charlesby s'applique aussi bien globalement que localement. Nous pouvons donc appliquer les relations (2), (3) et (4) et tracer les courbes des figures 6 , 7 et 8.

Cependant, malgré la constance de $E_{\mathrm{d}}$, on constate que la loi de Charlesby ne s'accorde pas avec l'expérience. En effet :

1. En traçant $1 \overline{M n}, f=f(Q)$ figure 6 , on voit que les courbes expérimentales peuvent être approximées par des droites qui ne vérifient pas $1 / \overline{M n}, f=1 / \overline{M n}, o$ à dose nulle $(1 \overline{M n}, f=1 / \overline{M n}, o$ pour une dose proche de $\left.2 \times 10^{13} \mathrm{el} / \mathrm{cm}^{2}\right): 1 / \overline{M n}, f=-11,4 \times$ $10^{-6}+82,62 \times 10^{-20} \cdot Q$.

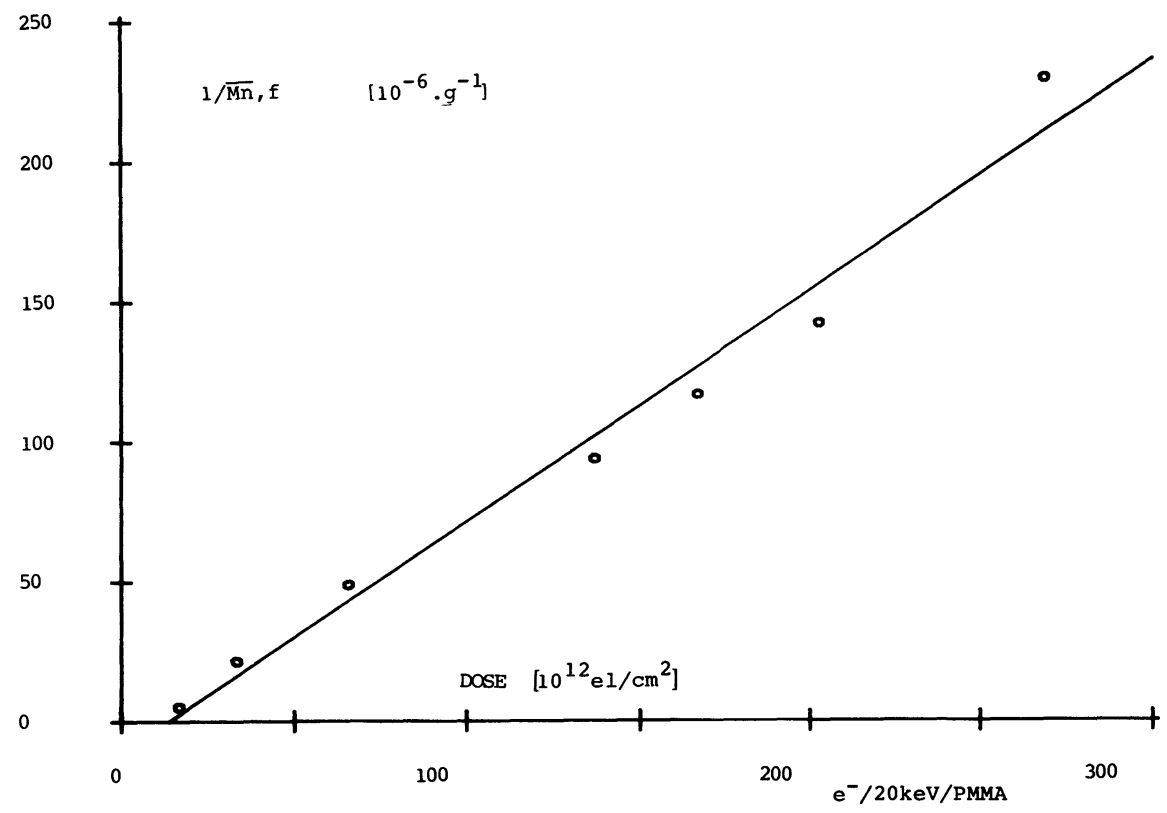

Fig. 6. - Trace de $1 \overline{M n}, f=f$ (DOSE).

[Curve for $1 / \overline{M n}, f=f$ (DOSE).] 


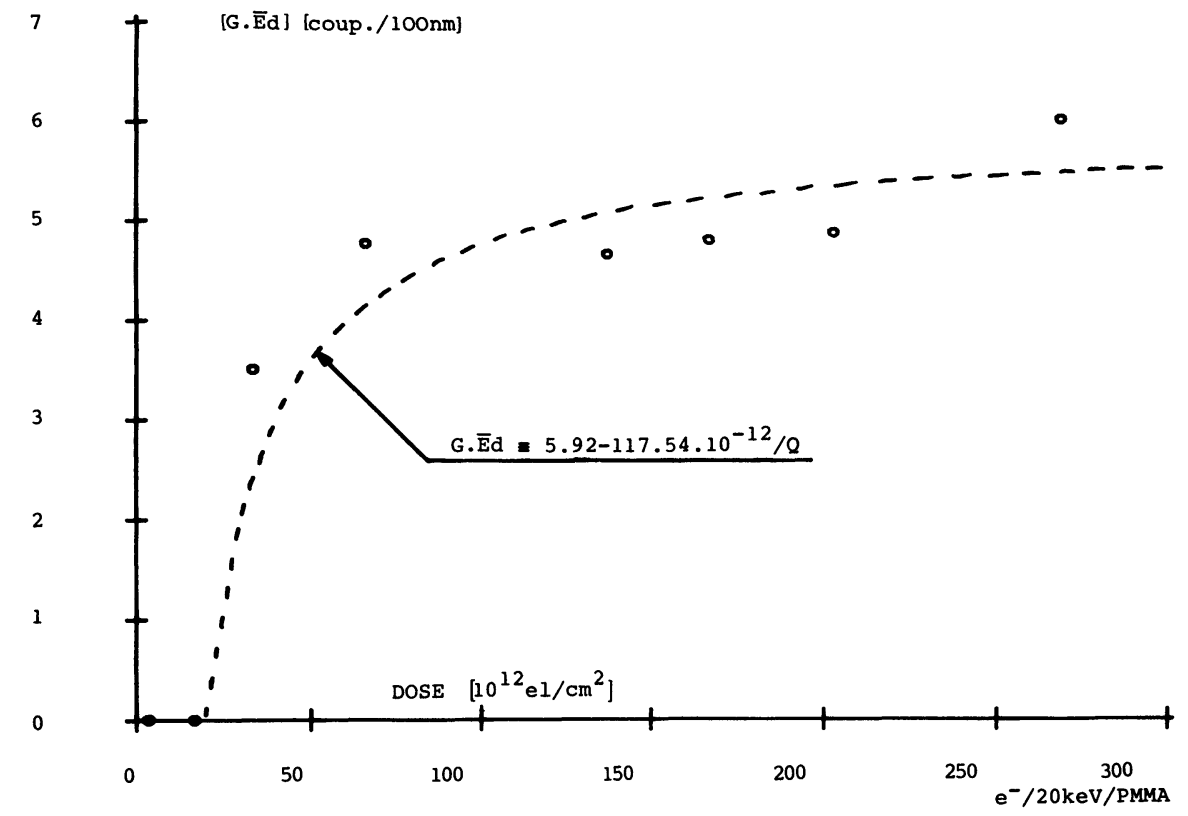

Fig. 7. - Calcul de $\left[G . \bar{E}_{\mathrm{d}}\right]=f(Q)$ avec (3).

[Calculated curve for $\left(G . \bar{E}_{\mathrm{d}}\right)=f(Q)$ from (3).]

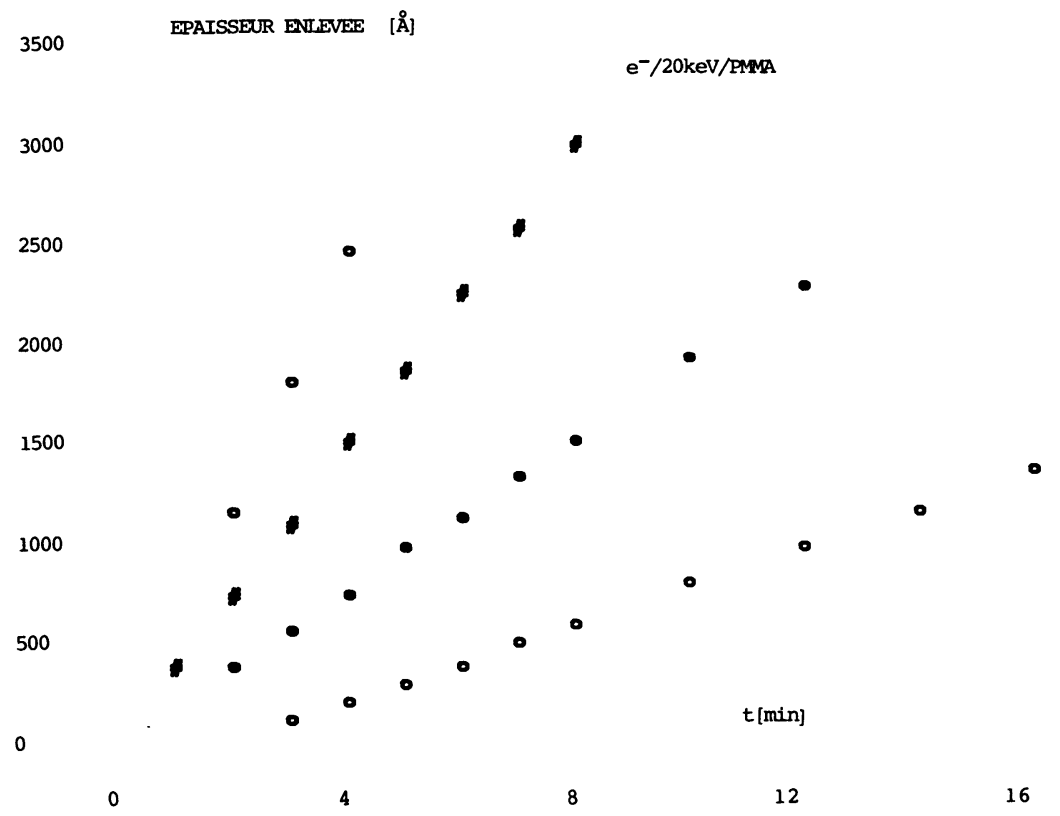

Fig. 8. - Points expérimentaux et courbes théoriques calculées avec l'équation de Charlesby.

[Experimental points and theoretical curves obtained using Charlesby's law.]

2. On observe figure 7 que $\left(G . \bar{E}_{\mathrm{d}}\right)$ et par suite $G$ dépendent fortement de la dose. Les points calculés admettent même une interpolation du type : $\left(G \cdot \bar{E}_{\mathrm{d}}\right)=$ $a-b / Q$.

3. Nous avons calculé les courbes de développement à l'aide de l'équation (4) en fixant $G=2,6$. On voit figure 8 que la loi ne permet pas de relier les points expérimentaux de la figure 2 .
Ainsi, même dans le cas où $E_{\mathrm{d}}$ est constant, nous mettons en évidence trois résultats expérimentaux en contradiction avec la loi de Charlesby. Nous lèvẹrons cette contradiction en partie II.

3.2 Application au Cas IONiQUe $\left(\mathrm{H}^{+} / 50 \mathrm{keV} / e>R_{\mathrm{p}}\right)$. - Les courbes de développement sont tirées de [7]. Elles concernent un PMMA $(\overline{M n}, o \simeq 244600)$ irra- 
dié par des protons ayant une énergie incidente de $50 \mathrm{keV}$ et développé dans un mélange de MIBK et d'Isopropanol (MIBK/IPA $=1 / 1)$ après un recuit de $30 \mathrm{~min}$ à $170^{\circ} \mathrm{C}$. Nous avons déterminé expérimentalement la loi de dissolution et nous avons établi une loi du même type que celle de Greeneich [5] dont les paramètres sont :

$$
\begin{aligned}
& A=1,5 \AA / \mathrm{min} \\
& B=91,55 \times 10^{6} \\
& \alpha=1,2 .
\end{aligned}
$$

L'épaisseur initiale, environ $10000 \AA$, est supérieure au parcours moyen projeté des particules $(7) ; R_{\mathrm{p}} \simeq$ $7000 \AA ̊$. L'énergie déposée n'est donc pas constante le long de l'épaisseur irradiée. Par conséquent, $\overline{M n}, f(x)$ et $V_{\mathrm{d}}(x)$ ne sont pas constantes et les courbes de développement ne sont pas linéaires (Fig. 3).

A partir des courbes de développement, nous avons calculé $\left(G . E_{\mathrm{d}}\right)(x)$ avec (3). Les courbes obtenues figure 4 ont été approximées figure 9 par des droites avec un bon coefficient de corrélation. Ces droites se rejoignent toutes au point $R_{\mathrm{c}}\left({ }^{3}\right)$.

Comme prévu, la loi de Charlesby n'est pas vérifiée. En effet :

- On devrait théoriquement observer figure 9. une courbe unique indépendante de la dose. Or on observe une courbe différente pour chaque dose, ce qui

$\left({ }^{3}\right)$ Remarque : Nous avons noté ce point, $R_{c}$, car il est le lieu moyen où les effets de la dégradation cessent d'être chimiquement observables. C'est donc le lieu où l'énergie effective s'annule. signifie que $\left(G . E_{\mathrm{d}}\right)(x)$ et par suite $G$ dépendent de la dose.

- Etant donné qu'on n'a pas une expression de $\left(G . E_{\mathrm{d}}\right)(x)$ unique quelle que soit la dose, on ne peut pas calculer de courbe de développement, $x(t)$, avec l'équation (4).

- On constate figure 10 , que les courbes $1 / \overline{M n}, f(x)=f(Q)$ calculées pour quelques valeurs de $x$ ne vérifient pas $1 / \overline{M n}, f(x)=1 / \overline{M n}, o$ à dose nulle extrapolée $(1 / \overline{M n}, f(x)=1 / \overline{M n}$, o pour une dose proche de $6 \times 10^{12}$ ions $\left./ \mathrm{cm}^{2}\right)$.

\section{Conclusion.}

Nous avons proposé une méthode expérimentale de calcul du profil du taux linéaire de dégradation à partir des courbes de développement.

Cette méthode nous a permis, en traitant deux exemples, de mettre en évidence le désaccord de la loi de Charlesby avec l'expérience. Nous proposons en partie II une modification simple de cette loi.

\section{Notations.}

$x(t)$ : Courbe de développement : épaisseur de résine enlevée au bout du temps « $t$ » de développement ( $\AA$ ).

$V_{\mathrm{d}} \quad$ : Vitesse de dissolution de la couche de résine $(\AA / \mathrm{min})$.

$A, B, \alpha:$ Constantes de la loi de dissolution de Greeneich [5] ( $\AA / \mathrm{min})$.

$\overline{M n}, o:$ Masse moléculaire moyenne en nombre de la résine non irradiée $(\mathrm{g})$.

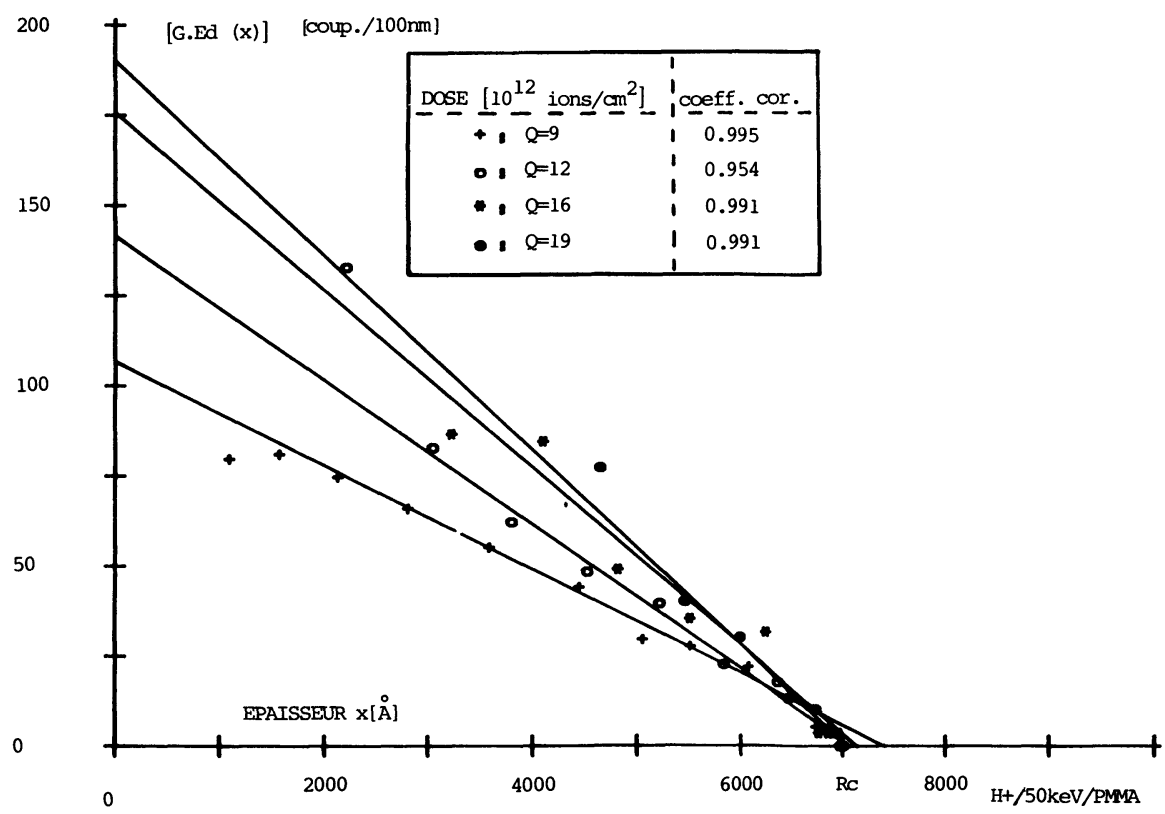

Fig. 9. - Taux linéaire de dégradation calculé avec (3).

[Linear scission yield from (3).] 


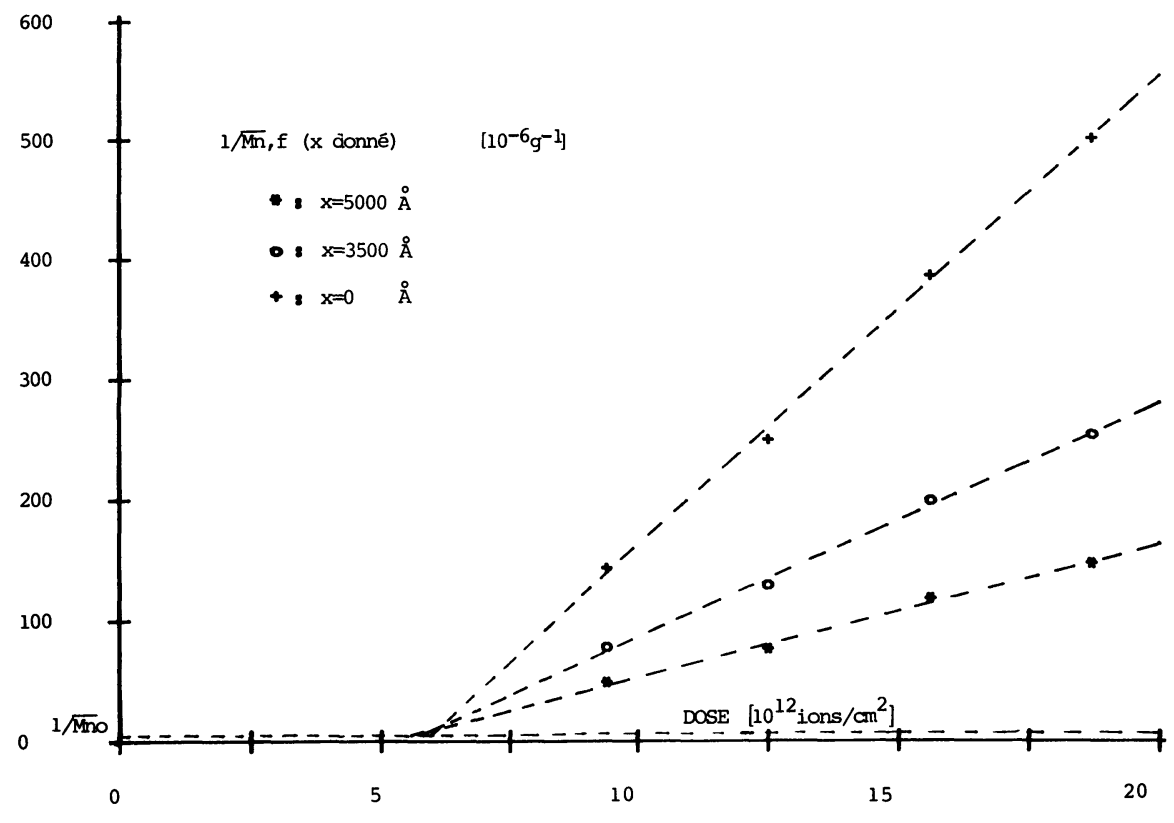

Fig. 10. - Expression de $1 / \overline{M n}, f(x)=f(Q)$ pour trois valeurs de $x . \mathrm{H}^{+} / 50 \mathrm{keV} / \mathrm{PMMA}$.

[Curves for $1 / \overline{M n}, f(x)=f(Q)$ calculated for three values of $x$.]

$\overline{M n}, f:$ Masse moléculaire moyenne en nombre des chaînes fragmentées (résine positive) $(\mathrm{g})$.

$\rho \quad$ : Masse volumique du polymère $\left(\mathrm{g} / \mathrm{cm}^{3}\right)$.

$N_{\mathrm{A}} \quad$ : Nombre d'Avogadro.

$G \quad$ : Taux de cou ure pour $100 \mathrm{eV}$ absorbés (coup/100 eV).

$E_{\mathrm{d}} \quad$ : Energie déposée par unité de longueur par un ion $(\mathrm{eV} / \mathrm{nm})$.
$Q \quad:$ Dose en flux de particules (part. $/ \mathrm{cm}^{2}$ ).

$R_{\mathrm{p}} \quad$ : Parcours moyen projeté des particules. Valeur moyenne de la profondeur de pénétration des particules, projetée sur l'axe du faisceau incident.

d'être chimiquement observables. Lieu où l'énergie effective s'annule. 